\title{
Digital Human Modeling for Indian Anthropometry
}

\author{
Abira DASGUPTA ${ }^{a}$, Bharat VIJAYARAGHAVAN ${ }^{a}$, N. R. RAJHANS ${ }^{a}$, \\ D.D. KULKARNI ${ }^{\mathrm{b}}$, A.V. MANNIKAR ${ }^{\mathrm{b}}$

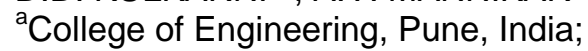 \\ ${ }^{\mathrm{b}}$ Automotive Research Association of India
}

\begin{abstract}
Traditionally, computer manikins are used to convert the anthropometric data into more usable form for industrial application. Several product design software offer the option for creating digital manikins, for example, RAMSIS, CATIA, UGS, DELMIA, etc. These software make use of various boundary manikin methods such as multi percentile approach, central region boundary approach, Principal Component Analysis, etc. to create digital models.

The anthropometric data for Indian population has been largely missing. Efforts have been undertaken by various academic institutions in India to collect this data over last several years. With the boom in the automotive industry, demand for collection of statistically robust anthropometric data has arisen from different segments of the industry. To meet this requirement, the Automotive Research Association of India (ARAI) undertook a 3D whole body scanning survey in India. In this survey, data of more than 5600 volunteers has been collected. This is the first 3D whole body survey conducted in India.

The research aims at reviewing different concepts of digital human modeling and applying a suitable manikin generation technique to the anthropometric data generated by the SIZE INDIA survey. No such effort has been made before to convert this data into digital human models, in India.
\end{abstract}

Keywords: Digital human modeling, anthropometry, manikins

\section{Introduction}

The study of anthropometry is the study of human body measurements to assist in understanding human physical variations and aid in anthropological classification. Anthropometry refers to the measurement of the human individual. It has been used for the purposes of understanding human physical variation. Anthropometry plays an important role in industrial design, clothing design, ergonomics and architecture where statistical data is used to optimize products. Ergonomic design uses anthropometric data to ensure that the equipment and surrounding environment fits the person. For this purpose it is important to have details of dimensions of appropriate body parts.

3D anthropometric data, obtained using 3D imaging technologies, provide detailed shape information. In addition, traditional measurements can also be extracted from the 3D models. Therefore, 3D anthropometric data offers an opportunity to improve the quality of the design models and, at the same time, maintain the simplicity of the traditional design schemes where key body dimensions are used.

The principle that a product should be comfortable to use for the user is the most universally employed concept in ergonomics. If a product is to be used by only one user, careful measurements of that person's body will yield appropriate dimensional specifications for that product. However, in various fields, where a single product must accommodate a large percentage of the population, the constraints on the design values are usually imposed by the desire to accommodate a sufficient range of the population on the anthropometric measures [1].

\section{Objective: Defining Digital Human Models (DHMs) for Indian anthropometry}

An anthropometric model is characterized by the exterior skin model that gives it a realistic appearance as well as by the interior skeletal model. The task of this interior model is to represent all postures and motion functions of the human body using as few joints as possible. However, due to recent improvements in computing capabilities, this restriction disappears more and more, favoring a realistic representation of the functionality of the skeleton. Nowadays even the skeleton can be displayed in a realistic form. The interior model serves as a framework for the exterior features connected to it. Whether they are statically or elastically connected is determined by mathematical algorithms.

*kulkarni.psl@araiindia.com; +91- 99752-68350; www.araiindia.com 
A special challenge for anthropometric human models is the correct representation of body parts, focusing on mutability and reach spheres. A simple transfer of values from anthropometric tables generated by conventional measuring methods does not yield the desired results. Most of the models developed in the past used anthropometric measures from tables which had to be adapted to the necessities of the DHMs. In fact, conventional anthropometric data are derived from bone to bone measurements and therefore need to be modified for existing models [2].

Three-dimensional manikins are also known as Digital Human Models. These are software representations of humans that enable designers to visualize the effectiveness of a design before a physical prototype is constructed. The DHM programs can be used to assess many design concerns. For example, automotive companies can utilize DHM to examine if the current seat adjustability will allow a wide range of users to reach all of the needed controls. Being able to do all of this on a computer rather than using a physical prototype, results in faster, higher quality, and more accessible designs that also lower cost. Various software like CATIA, UGS, DELMIA, JACK, RAMSIS, SAFEWORK can be used to create digital manikins. Digital human models make use of the boundary manikin concept, where statistically extreme cases are used to represent the less extreme cases of a certain population.

Various studies have been undertaken related to Indian anthropometry in fields like history and classics, nutrition, agriculture machinery design, and physical anthropology [3,4,5]. An anthropometric database created by the DRDO (Defence Research and Development Organisation) consisted of anthropometric dimensions of mainly personnel from the Indian armed forces. This database was created to be used in the design of clothing and personal warfare equipment. Another database of the anthropometry of Indian women was generated to be used in the ergonomic designing of workspaces, seats, controls, etc. for the reference population. This study consisted only of the Indian women aged $12-20[6]$.

Recently, the Automotive Research Association of India has launched a project on the anthropometric size measurements of the Indian population, using 3-D whole body scanner technology. Under this project, anthropometric measurements of around 5600 people in India have been taken. This technology has been used in India for the first time to assist the taking of automatic anthropometric size measurements of human subjects in a very short duration. A digitized 3-D image of human body is created and a post processing software helps in extracting more than 150 different dimensions from the scanned image. Such anthropometric data is of great use for various industrial design activities like automotive interior design, occupant cabin design, readymade garment design, shoes and helmet design, 3-D computer games/animation and many more applications.

\section{Literature Review}

A special challenge for anthropometric human models is the correct representation of body parts that focus on mutability and reach spheres, and represent entire population of users by a family of manikins referred to as boundary manikins. A simple transfer of values from anthropometric tables generated by conventional measuring methods does not yield the desired results [2]. Various mathematical techniques are used in the generation of boundary manikins. Some of these are the percentile approach, Principal Component Analysis (PCA), and the confidence ellipse method.

\subsection{Percentile approach}

In the percentile approach, the key dimensions are set to a specific percentile value. This approach forms an imaginary square accommodation region (for two dimensions) formed by the respective confidence intervals for each dimension separately. The results obtained through univariate analysis when compared with those of multivariate analysis, demonstrate that application of univariate analysis to a multivariate problem leads to misleading results and poor estimates of accommodation. It has been found that approximately only $85 \%$ of the individuals are enclosed by the square area for a $90 \%$ desired accommodation level (dimensions are set between 5 percentile and 95 percentile) [7].

\subsection{Principal component analysis}

Another technique called Principal Component Analysis (PCA) identifies the direction of maximum variance amongst a set of dimensions [8]. It can be used to identify the underlying basis of variability within the anthropometric factors considered. PCA re-expresses data along new orthonormal bases [9]. The first few principal components capture the maximum variability if the data are highly correlated. It is a data reduction procedure that can greatly simplify the use of a test sample for accommodation or design studies by reducing the number of dimensions of a hyper-ellipsoid. 
Boundary case methods often use PCA which makes it possible to reduce the dimensions of the problem without much loss of the variance of the analyzed data. It includes manual analysis at the critical stage of reducing dimensions.

\subsection{Confidence Ellipse Method}

The confidence ellipse method is based on the idea that the people imposing the least design requirements are located in the centre of the entire population distribution. In the one dimensional situation the optimum population subset is contained in an interval centered at the mean value and containing the required percentage of the entire population. The optimum test individuals are the people located at the end points of the interval. The above idea can be extended to multi-dimensional case, where the simultaneous probability distribution of the key variables has an elliptical shape. The size of the ellipse can be varied to accommodate any desired percentage of the entire population [10]. This method is used for defining appropriate boundary manikins according to the selected key dimensions under desired accommodation level, which is represented by the confidence region.

The confidence ellipse method makes use of the eigenvalues and eigenvectors of the covariance matrix to determine the confidence ellipsoid and the boundary manikins. The lengths of the axes of the ellipsoid are proportional to the eigenvalues, and the eigenvectors give the directions of these axes. The boundary manikins obtained by this method are located at the end points of each of the axes of the confidence ellipsoid, and one at the centre of the ellipsoid i.e. mean. Thus, the number of boundary manikins is given by -

$$
n=2 \cdot p+1
$$

\section{Methodology}

The Indian anthropometric data of only male population was used for analysis. The various parameters relevant to vehicle seat accommodation are listed in table 1. Figure 1 shows some of the seat design parameters and the corresponding shortlisted anthropometric parameters.

Table 1. Design parameters and corresponding anthropometric measurements.

\begin{tabular}{|l|l|}
\hline Design Parameter & Anthropometric measurement \\
\hline Cushion width & Hip breadth (sitting) \\
\hline Cushion length & Buttock-popliteal length \\
\hline Seat height & Popliteal height \\
\hline Backrest width & Biacromial breadth \\
\hline Backrest height & Shoulder height (sitting) \\
\hline Head clearance & Sitting height erect \\
\hline Vision & Eye height (sitting) \\
\hline Reach & Shoulder-point dactylion height, Elbow grip length, Forearm fingertip length \\
\hline Steering clearance & Knee height (sitting) \\
\hline Body dimension & Stature, Shoulder height (standing), lliac spine height (standing) \\
\hline
\end{tabular}



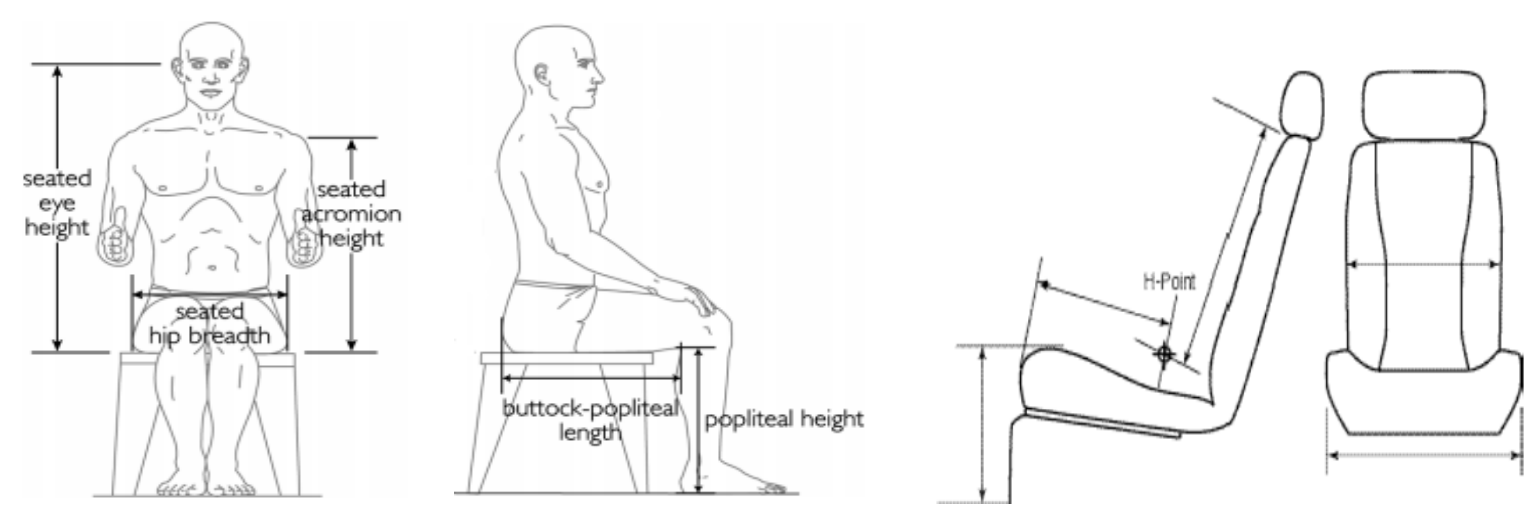

Fig. 1. Seat parameters and related anthropometric dimensions.

This data of the shortlisted parameters was converted into their z-scores, since this helps in giving each distribution the same significance in the calculations [11]. PCA was applied to this standardized data. MATLAB® software was used for the computations and the results obtained are shown in figure 2. Figure 2 shows the variances in data accounted for by the principal components, in a cumulative order, starting with the principal component accounting for the maximum variance and with subsequent principal components with decreasing variability. The results show that, in order to achieve 90\% accommodation level (variance in the data), 7 principal components have to be considered.

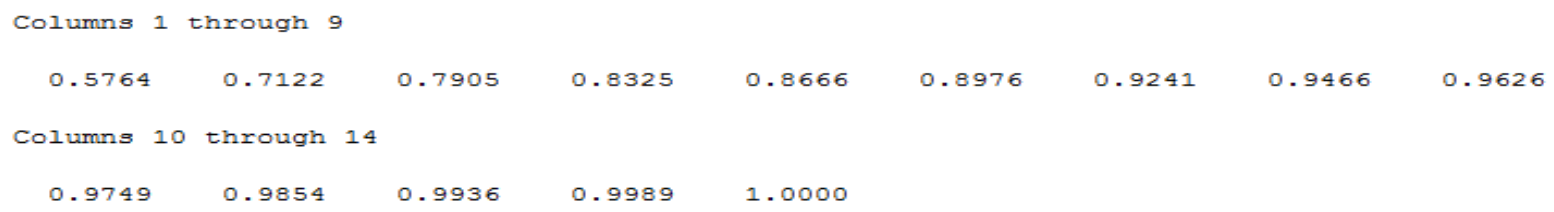

Fig. 2. Cumulative variances for the fourteen principal components.

The shortlisted anthropometric dimensions were classified into independent and dependent parameters, shown in table 2, and the dependent parameters can be calculated by regression. The root mean square error values (RMSE) are also listed in table 2, and they were found to be within reasonable limits. Hence, the regressed values can be considered to be fairly accurate.

Table 2. Dependent and independent parameters.

\begin{tabular}{|l|l|l|}
\hline \multicolumn{1}{|c|}{ Parameters } & \multicolumn{1}{|c|}{ Derived from } & $\begin{array}{l}\text { Root Mean } \\
\text { Square Error }\end{array}$ \\
\hline Iliac spine height (standing) & Stature & $3.37 \%$ \\
\hline Shoulder height (standing) & Stature & $1.22 \%$ \\
\hline Knee height (sitting) & Popliteal height & $3.07 \%$ \\
\hline Elbow grip length & Forearm fingertip length & $3.69 \%$ \\
\hline Shoulder Point dactylion height & Forearm fingertip length & $3.43 \%$ \\
\hline Eye height (sitting) & Shoulder height (sitting) & $2.73 \%$ \\
\hline Sitting height erect & Eye height (sitting) & $1.75 \%$ \\
\hline
\end{tabular}


After choosing the seven independent dimensions, PCA was performed on this data. Figure 3 shows the eigenvalues and cumulative variances for the seven principal components. It can be seen that, even for seven basic parameters, five principal components have to be considered if 90 percent accommodation level is needed. The eigenvalues indicate the magnitude of variability for the corresponding principal component.

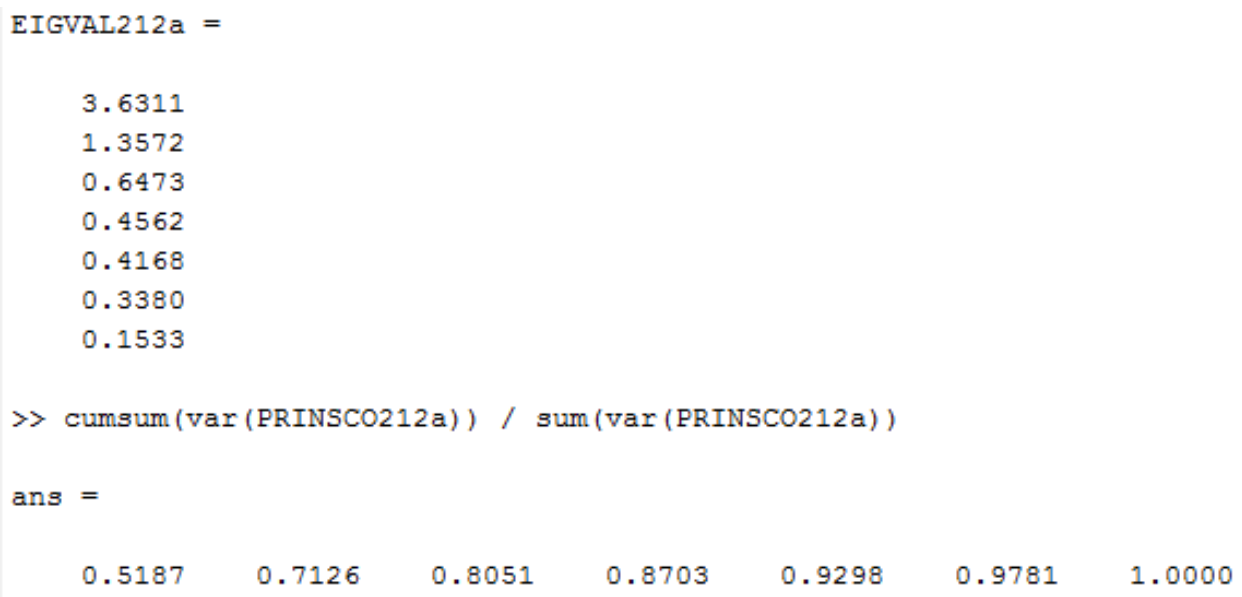

Fig. 3. Eigenvalues and cumulative variances accounted for by the principal components.

In case of three principal components (3-dimensional space) the boundary manikins that have to be taken into account are as follows - two at the extremes of each axis $(3 \times 2=6)$, four on each plane ( 3 $\times 4=12)$, and one in each quadrant $(8 \times 1=8)$. Thus, the total number of boundary manikins is 26 [12]. Using the same principle, for five principal components (5-dimensional space), the number of manikins to be considered for a given design application is equal to 62. Creating so many manikins is not pragmatic. Hence, the confidence ellipse method was applied to the seven independent parameters listed in table 2. Using this method, the number of manikins can be calculated by equation 1 , and is equal to 15 .

$$
\begin{aligned}
& 0.1533 \\
& 0.3380 \\
& 0.4168 \\
& 0.4562 \\
& 0.6473 \\
& 1.3572 \\
& 3.6311
\end{aligned}
$$

Fig. 4. Eigenvalues of seven parameters.

Figure 4 shows the eigenvalues of the covariance matrix of the data. These eigenvalues determine the lengths of the axes of the confidence ellipse. The ellipsoid whose axes are defined by the root of eigenvalues (magnitude of length of axes) and eigenvectors (direction) represent a confidence region of approximately $39.4 \%$. To obtain desired confidence level, the axes have been scaled up by a factor which is calculated using chi squared distribution and desired probability level for the confidence region [10].

Values of the shortlisted variables for each boundary manikin, $\mathrm{M}_{\mathrm{i}}$, which are defined as the points at the ends of each axis (one at the positive end and the other at the negative end of the axis) were calculated. The standardized scores of the dimensions of the key measurements of the 15 boundary manikins are shown in figure 5. 


\section{Results}

\begin{tabular}{|c|c|c|c|c|c|c|c|c|c|c|c|c|}
\hline \multicolumn{13}{|c|}{ MQNNRINYGLLESZVALLIES } \\
\hline$M 12$ & M22 & M31 & M32 & M41 & M42 & M51 & M/52 & MG6 & $M 62$ & M71 & M72 & Mmean \\
\hline $0.3212 \cdot 0.3212$ & $0.8014 \cdot 0.00014$ & -0.309 & 10.3091 & .15529 & 1.5529 & 0.1342 & -0.1342 & 17981 & . 1.798 & 2348 & 1.23481 & \\
\hline $0.4104-0.4104$ & $.16515 \quad 1.655$ & -0.2468 & 80.2468 & .0.0678 & 0.0678 & 0.2469 & -0.2669 & 0.5138 & -0.5138 & 2.9548 & $8 \cdot 2.9548$ & \\
\hline -1.284 1.2284 & $.0 .116 \quad 0.116$ & 0.127 & $1 \cdot 0.1271$ & .0 .0352 & 0.0352 & -0.6592 & 0.6592 & 0.5325 & 0.5325 & 3.1615 & $5-3.1615$ & \\
\hline $0.0231 \cdot 0.0231$ & $0.1091 \cdot 0.1091$ & 1.420 & $1 \cdot 1.4201$ & .0 .6042 & 0.6042 & 1.1262 & .11262 & $-2,1231$ & 2.1231 & 1.9614 & $4 \cdot 1.9614$ & \\
\hline $0.5187-0.5187$ & $0.3997-0.3997$ & 0.367 & $1 \cdot 0.3671$ & 0.4931 & -0.4931 & .207 & 207 & .0 .939 & 0.939 & 2.4584 & $4-2.4584$ & \\
\hline 0.05040 .0504 & $0.3669-0.3669$ & .16200 & 61.6206 & 0.1198 & -0.1198 & 0.5786 & 0.5786 & .2233 & 2233 & 1.9799 & 5.19795 & \\
\hline $0.1607-0.1607$ & $0.6101 \cdot 0.66101$ & 0.242 & 4.02424 & 15627 & .15627 & 1.7735 & .17735 & 1.4677 & .14677 & 23637 & 7.23637 & \\
\hline
\end{tabular}

Fig. 5. Z scores of the 7 parameters of the 15 manikins.

Figure 5 shows the z-scores of the seven parameters for a total of fourteen manikins at the end points of the axes of the confidence ellipse, and for one manikin at the centre of the ellipse. The manikins at the ends of the axes are given by Mij: $i=1,2, \ldots, p(p=$ number of parameters) and $j=1,2$ (representing the two end points of each axis). The z-score values for the mean manikins are zero, and the values of the manikins at the end points of the axes are mirrored about the centre i.e mean.

The actual dimensions of the manikins are obtained back from the standardized scores by multiplying the above values by the respective standard deviations and then adding their respective mean values. The dimensions of the manikins highlighted in blue in figure 6 (independent parameters) were calculated by the confidence ellipse method, and those highlighted in green were computed by the regression equations given earlier.

Thus, dimensions of 14 parameters of 15 different manikins have been found. These manikins are boundary manikins, which are statistically extreme cases that accommodate a big part of the less extreme population.

\begin{tabular}{|c|c|c|c|c|c|c|c|c|c|c|c|c|c|c|c|}
\hline MANKINVALUES & M11 & M12 & M21 & M22 & M31 & M32 & M41 & M42. & M51 & M52 & M61 & M62 & M71 & M72 & Mmean \\
\hline poplitea | height & 44.1731 & 1. 42.6092 & 45.3421 & 41.4403 & 42.638 & 1844.1436 & 39.611 & 47.1714 & 43.7178 & 843.0646 & 47.7682 & 239.0142 & 49.1073 & 337.6751 & 43.3912 \\
\hline forearm.tingertip lenghth & 48.2372 & . 46.3557 & 43.5104 & 51.0825 & 46.730 & 1847.8622 & 47.141 & 47.4519 & 47.8625 & 546.7304 & 48.4742 & 246.1187 & 54.0703 & 340.5227 & 47.2965 \\
\hline stature & 159.694 & 175.049 & 166.582 & 168.161 & 168.23 & 6166.507 & 167.132 & 2167.611 & 162.887 & 7171.856 & 170.994 & 4163.749 & 188.882 & 12145.86 & 167.371 \\
\hline biaccomial breadth & 37.115 & 37.03 & 37.273 & 36.872 & 39.682 & 634,4624 & 35.962 & 238.183 & 39.1425 & 535.0025 & 33.1703 & 340.9747 & 40.6775 & 533.4675 & 37.0725 \\
\hline shoulder heightsitting & 60.6161 & 57.4507 & 60.2529 & 57.8139 & 60.153 & 1757.9131 & 60.5381 & 157.5287 & 52.717 & 765.3498 & 56.1683 & 361.8985 & 66.5348 & 851.532 & 59.0334 \\
\hline hip breadth sitting & 35.4475 & 35.7743 & 36.7994 & 34.4224 & 30.361 & 640.8602 & 35.9989 & 935.2229 & 37.485 & 133.7366 & 28.378 & 842.8438 & 42.0227 & $7 \quad 29.199$ & 35.6109 \\
\hline buttock popliteal lenggh & 47.893 & 46.8873 & 49.2987 & 45.4815 & 48.148. & 6646.6316 & 52.2788 & 842.5015 & 51.061 & 4.43 .7188 & 51.9818 & 842.7984 & 54.7849 & 939.9953 & 47.3901 \\
\hline knee height sitting & 53.7482 & 52.3759 & 54.774 & 51.3501 & 52.401 & 1853.7223 & 49.745 & 556.3792 & 53.3488 & 652.7755 & 56.9029 & 949.2213 & 58.0779 & 948.0462 & 53.0621 \\
\hline iliac spine heightstanding & 82.7486 & 92.2274 & 87.0007 & 87.9753 & 88.021 & 1986.9541 & 87,3402 & 287.6358 & 84.7199 & 590.2565 & 89.7244 & 485.2516 & 100.767 & 74.2092 & 87.488 \\
\hline elloow grip length & 37.0676 & 35.6747 & 33.5684 & 39.174 & 35.952 & $14 \quad 36.79$ & 36.2561 & 36.4863 & 36.7902 & 235.9521 & 37.2431 & 135.4993 & 41.3858 & 831.3565 & 36.3712 \\
\hline eve heights sitting & 76.3894 & 73.2418 & 76.0283 & 73.603 & 75.929 & 673.7016 & 76.3119 & 1973.3193 & 68.5348 & 681.0966 & 71.9666 & 677.6647 & 82.275 & 567.3562 & 74.8156 \\
\hline sitting height erect & 87.5223 & 84.5161 & 87.1774 & 84.861 & 87.083 & 1284.9552 & 87.4483 & 1384.5901 & 80.0202 & 292.0182 & 83.2981 & 188.7403 & 93.1437 & 778.8947 & 86.0192 \\
\hline shoulder height standing & 133.085 & 146.42 & 139.067 & 140.438 & 140.50 & 14139.002 & 139.545 & 15139.961 & 135.858 & 8143.647 & 142.899 & 9136.607 & 158.433 & 13121.073 & 139.753 \\
\hline shoulder point dactylion len & 76.2925 & 73.7156 & 69.8187 & 80.1894 & 74.229 & 1275.7788 & 74.7911 & 1175.217 & $75.779:$ & 374.2288 & 76.6171 & 173.391 & 84.2815 & 565.7266 & 75.004 \\
\hline
\end{tabular}

Fig. 6. Actual values of the parameters of the manikins.

These values were used to generate manikins in CATIA V5, and they have been shown graphically in figure 7 in various postures. These manikins can be integrated in various virtual environments. The different postures can be used as an aid in the design of diverse products. Thus, the manikin family is applicable not only to vehicle accommodation, but also to a number of different designs, making repeatability of use possible. 

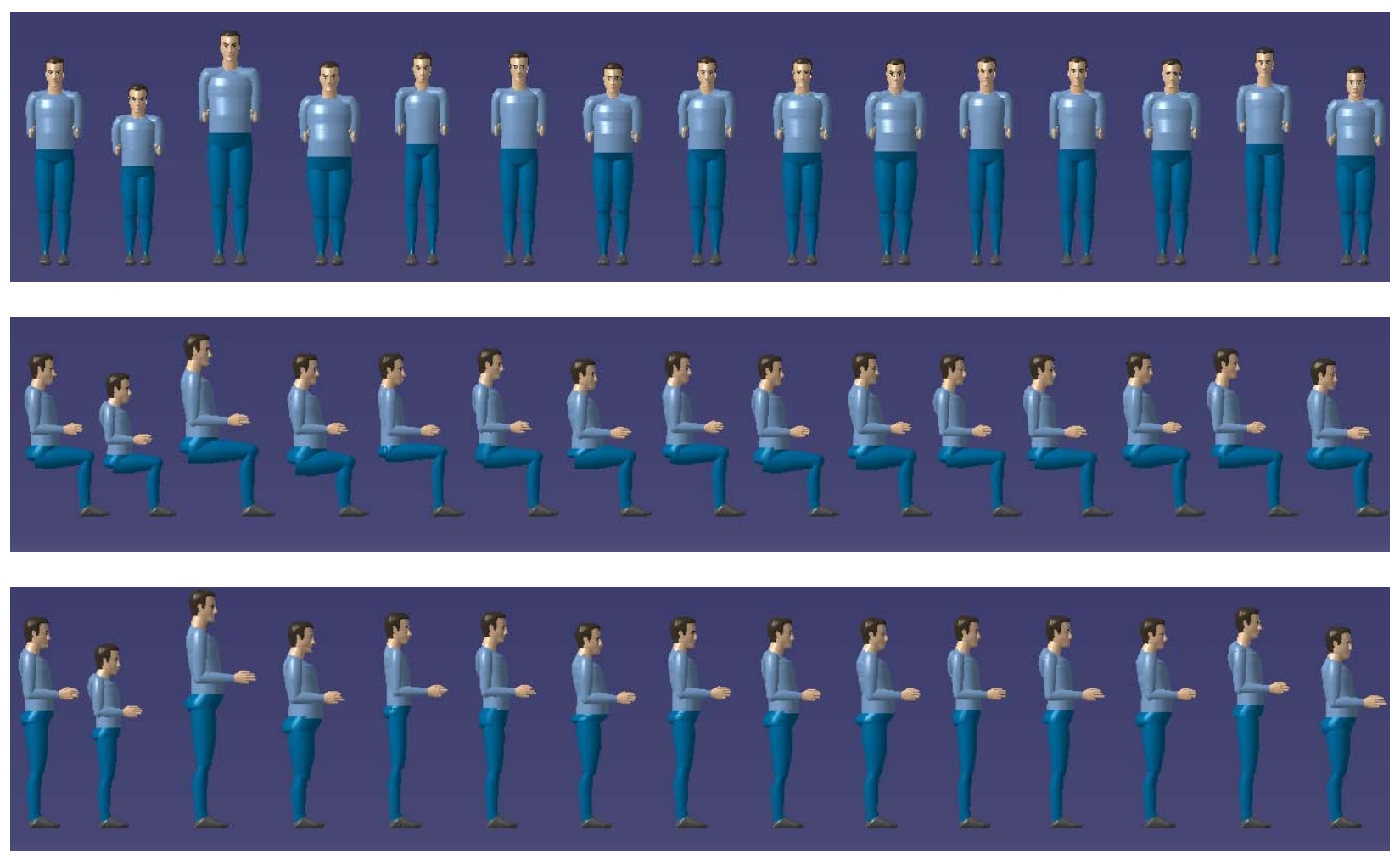

Fig. 7. Visual representation of the manikins in CATIA V5.

\section{Conclusion}

This paper explores various methods which make it possible to represent the Indian anthropometric data by a set of boundary manikins for the purpose of vehicle seat accommodation. The data considered was for the male population aged between 18 and 70. A manikin can be defined better with more number of parameters. However, with the increase in the number of parameters used for analysis, the number of boundary manikins generated by PCA may be overwhelming. In the analysis done in this paper, using the confidence ellipse method resulted in the creation of just 15 manikins, as opposed to 62 manikins which would have to be generated using PCA. Since it is crucial to keep the boundary cases to a minimum, the confidence ellipse method was used to reduce the number of manikins considerably, without any loss in the variability. CATIA was used to generate this set of manikins for the purpose of visual comparison.

\section{Acknowledgement}

We would like to acknowledge the support of Mr. Kannan Nair from CSM Software Pvt. Ltd. for providing licensed access to CATIA V5, and thus, for creation of the virtual manikins. The support of the Automotive Research Association of India, for providing the Indian anthropometric data, is also gratefully acknowledged. 


\section{References}

1. Reed, M. (2000): Survey of Auto Seat Design Recommendations for Improved Comfort, Technical Report: UMTRI-94-6, University of Michigan Transportation Research Institute.

2. Duffy, V., G.: Handbook of Digital Human Modeling: Research for Applied Ergonomics and Human Factors Engineering. Boca Raton: CRC Press.

3. L.P. Gite, B.G. Yadav, Anthropometric survey for agricultural machinery design: An Indian case study, Applied Ergonomics, Volume 20, Issue 3, September 1989, Pages 191-196, ISSN 00036870, 10.1016/0003-6870 (89) 90076-8.

4. Bisai, S., Bose, K., Khatun, A., Bauri, H. (2009): Age-Related Anthropometric Changes and Undernutrition among Middle Aged and Older Savar Tribal Females of Keonjhar District, Orissa, India. Journal of Life Sciences, Volume 1, Issue 1, Pages 21-26 (2009), ISSN 0975-1270.

5. Singh, R. D. (1974), Problems of techniques in anthropometric studies in India. Am. J. Phys. Anthropol., 40: 221-226. doi: 10.1002/ajpa.1330400209.

6. Defence Institute of Physiology \& Allied Sciences, Ministry of Defence, Govt. of India. (http://drdo.gov.in/drdo/labs/DIPAS/English/index.jsp?pg=Products.jsp).

7. Wirsching, H., Premkumar, S.(2007), "Statistical representations of human populations in ergonomic design," SAE Technical Paper 2007-01-2451, 2007, doi: 10.4271/2007-01-2451.

8. I. T. Jolliffe. Principal components analysis. Springer series in Statistics. Springer, 2004.

9. L. I. Smith. A tutorial on principal component analysis. http://kybele.psych.cornell.edu//edelman/Psych-465-Spring-2003/PCA-tutorial.pdf.

10. Bertilsson, E., Hogberg, D., Hanson, L., Wondmegagne, Y. (2011): Multidimensional consideration of anthropometric diversity. Proceedings of DHM 2011, First International Symposium on Digital Human Modeling, France, June 2011, ISBN 978-2-9539515-0-9.

11. Glenberg A, Andrzejewski M, (2007): Learning from Data: An Introduction to Statistical Reasoning, $3^{\text {rd }}$ edition. Routledge Academic, New York, ISBN 9780905849219.

12. Young, K., Margerum, S., Barr, A., Ferrer, M., et al., "Generation of Boundary Manikin Anthropometry," SAE Technical Paper 2008-01-2107, 2008, doi: 10.4271/2008-01-2107. 